\title{
Propriedades Psicométricas do Inventário de Fatores Psicológicos de Doenças Relacionadas ao Trabalho: Um Estudo com Trabalhadores de Frigoríficos
}

\author{
Romilda Guilland ${ }^{1}$ \\ Roberto Moraes Cruz ${ }^{2}$ \\ Erikson Kaszubowski $i^{2}$ \\ ${ }^{1}$ Comissão de Avaliação Psicológica do Conselho Regional de Psicologia, Cascavel, PR \\ ${ }^{2}$ Universidade Federal de Santa Catarina - UFSC, Florianópolis, SC
}

\begin{abstract}
Resumo
Este estudo avaliou as propriedades psicométricas do Inventário de Fatores Psicológicos em Doenças Relacionadas ao Trabalho (IP-T), com base em indicadores de validade de critério, validade de construto e fidedignidade. A amostra foi composta por 355 trabalhadores de indústrias de abate de suínos, aves e outros pequenos animais, da região oeste do Paraná. Foram também utilizados: o Self-Reporting Questionnaire (SRQ-20) e a Escala de Ansiedade e Depressão (HADS). As subescalas do IP-T se mostraram preditoras positivas da condição de afastamento por doença ocupacional, com sensibilidade e especificidade razoáveis. A matriz de correlação entre as escalas mostrou-se uniforme, com coeficientes entre 0,80 e 0,91 , sugerindo convergência entre as subescalas do IP-T e outras escalas, mas indicando fraco poder discriminativo. Os índices de fidedignidade das subescalas do IP-T mostraram-se satisfatórios: entre 0,95 (Ansiedade) e 0,78 (Habilidades Sociais).

Palavras-chave: transtorno mental, propriedades psicométricas, teste psicológico, doenças ocupacionais, frigoríficos
\end{abstract}

Psychometric Properties of the Psychological Factors of Deseases Related Work Inventory: A Study with Workers of the Meat Processing Industry

\begin{abstract}
This study assesses the psychometric properties of the Inventory of Psychological Factors in Work-Related Illnesses (IP-T), based on the following indicators: criterion validity, construct validity, and reliability. The sample consisted of 355 workers in the meatpacking industry of pork, poultry and other small animals in the west of the state of Paraná. The Self-Reporting Questionnaire (SRQ-20) and the Hospital Anxiety and Depression Scale (HADS) were also used. All sub-scales of the IP-T proved positive predictors of sick leave due to occupational disease, with moderate sensitivity and specificity. The correlation matrix between the scales was uniform, with coefficients between 0.80 and 0.91 , suggesting convergence between the subscales of the IP-T and other scales, but indicating a weak discriminative power. The reliability indices of the sub-scales of the IP-T proved satisfactory: between 0.95 (Anxiety IP-T) and 0.78 (Social Abilities).

Keywords: Mental disorders, psychometric properties, psychological test, occupational diseases, meatpacking industry
\end{abstract}

Propiedades Psicométricas do Inventario de Factores Psicológicos de las Enfermedades Relacionadas al Trabajo: Un Estudio con Trabajadores de Frigoríficos

\section{Resumen}

Se evaluaron las propiedades psicométricas del Inventario de Factores Psicológicos de Enfermedades Relacionadas al Trabajo (IP-T), con base en indicadores de validez de criterio, validez de constructo y fidedignidad. La muestra fue compuesta por 355 trabajadores de un frigórifico de cerdos, aves y otros pequeños animales de la región oeste de Paraná. Fueran también utilizados: el Self-Reporting Questionnaire (SRQ-20) y la Escala de Ansiedad y Depresión (HADS). Las subescalas de IP-T se mostraran predictores positivos de la condición de alejamiento del trabajo por enfermedad ocupacional con sensibilidad y especificidad razonables. La matriz de correlación entre las escalas se mostró uniforme, con coeficientes entre 0,80 y 0,91 , sugiriendo convergencia entre las subescalas de IP-T y otras escalas, pero indicando un débil poder discriminativo. Los índices de fidedignidad de las subescalas del IP-T se mostraron satisfactorios: entre 0,95 (Ansiedad) y 0,78 (Habilidades Sociales).

Palabras-clave: trastorno mental, propiedades psicométricas, test psicológico, enfermedades ocupacionales, frigoríficos

\section{Introdução}

No Brasil, a implementação da Norma Regulamentadora (NR-36) fez com que fosse lançado um novo olhar sobre a segurança e saúde no trabalho em empresas de abate e processamento de carnes e derivados, o que possibilitou melhoria dos processos de trabalho.
Porém, a indústria avícola ainda apresenta riscos ocupacionais relevantes, especialmente no setor de corte e pendura de aves, dentre os quais o trabalho intensivo, tarefas e ciclos repetitivos, que podem atingir o abate de 35 aves por minuto (Harmse, Engelbrecht, \& Bekker, 2016). O volume e a intensidade dos riscos ocupacionais nesse setor econômico contribuem decisivamente para 
a quantidade de afastamentos do trabalho e em danos à saúde dos trabalhadores, dentre os mais prevalentes, os distúrbios musculoesqueléticos (Pinetti \& Buczek, 2015) e os transtornos mentais (Ikedo \& Ruiz, 2015).

O transtorno mental é uma síndrome causada por perturbações clinicamente significativas, que reflete em uma disfunção nos processos biológicos, psicológicos e/ou de desenvolvimento subjacentes ao desenvolvimento mental (APA, 2013). Também é frequentemente associado ao sofrimento ou incapacidade significativa que interfere na vida social, profissional e, de modo geral, em todas as atividades desenvolvidas pelo trabalhador (Cruz, 2010).

Transtornos mentais são um problema de saúde pública, cujas as origens e formas de desencadeamento também se encontram no mundo do trabalho, mas nos países em desenvolvimento, os dados são escassos e restritos, seja do ponto de vista clínico ou epidemiológico (Cruz, 2010). Um dos principais fatores que favorecem a falta de informação precisa sobre transtornos mentais relacionados ao trabalho são os subdiagnósticos (Gonçalves, Stein, \& Kapczinski, 2008). Além disso, apesar do Nexo Técnico Epidemiológico Previdenciário (NTEP) ter sido implementado no Brasil em 2007, a subnotificação das doenças no Brasil ainda é uma realidade (Ikedo \& Ruiz, 2015).

No Brasil, a legislação vigente da Previdência Social (lei 8.213/91), pondera que o empregado que ficar afastado do trabalho durante 15 dias consecutivos, no $16^{\circ}$ dia, ele é encaminhado para realizar a perícia médica e receber o auxílio-doença pago pelo INSS. Para obter o auxílio-doença previdenciário, código B31, o empregado deve ter feito 12 contribuições para a Previdência Social e, no seu retorno, não há qualquer garantia de emprego. Mas, para receber o auxílio-doença acidentário, código B91, não está previsto qualquer período de carência, e, na sua volta ao emprego, o trabalhador terá garantida a sua permanência na empresa pelo período de 12 meses.

O NTEP indica a existência do nexo de causalidade entre a atividade laboral desenvolvida nas indústrias de abate e os transtornos mentais, porém, conforme os resultados da pesquisa realizada pelos pesquisadores do Instituto Fator Humano (2013) apontam, quando o trabalhador recebe o diagnóstico, este geralmente é referente ao benefício auxílio-doença previdenciário (B31), concedido aos segurados portadores de doenças comuns, diferentemente daquele relacionado ao trabalho, denominado de auxílio-doença acidentário (B91). Nesse caso, os subdiagnósticos geram problemas sociais e de proteção à saúde dos trabalhadores, uma vez que restringem direitos aos trabalhadores assegurados em lei, influenciam na busca e manutenção de tratamento aos agravos à saúde, decorrente do diagnóstico prescrito, assim como no processo de recuperação da saúde (Jaques, 2007; Walter, 2012).

Para realizar o diagnóstico de transtorno mental, existem instrumentos de rastreio que podem ser utilizados na avaliação clínica da população adulta, proporcionando além da detecção precoce, diagnósticos mais precisos. Dos 15 instrumentos referidos por Bolsoni e Zuardi (2015), apenas oito apresentam valores de confiabilidade e 10 validade de critério, concorrente e discriminante. Destes, apenas dois instrumentos foram validados no Brasil, o Dartmouth Primary Care Cooperative Research Network/World Organization of National Colleges (COOP/WONCA) e o Self-Reporting Questionnaire-20 (SRQ-20).

O SRQ-20 é um instrumento amplamente utilizado para suspeição diagnóstica de transtornos mentais comuns. A estrutura fatorial e consistência interna do SRQ-20 foram avaliadas em uma população de trabalhadores brasileiros, sendo que a proporção cumulativa de explicação da variância entre os trabalhadores estudados foi de 59,6\%. Esse resultado indicou que os quatro fatores extraídos (fator I - comportamento ansioso e depressivo, fator II - decréscimo de energia, fator III - sintomas somáticos, fator IV - humor depressivo), por meio da análise fatorial de correlações tetracóricas possuem um bom nível de explicação para a proporção de variância. Nesse caso, o instrumento foi considerado capaz de identificar fatores que, juntos, denotam características indispensáveis para o rastreamento do transtorno mental comum no âmbito ocupacional (Santos, Araújo, \& Oliveira, 2009).

O Inventário de Fatores Psicológicos para Doenças Relacionadas ao Trabalho (IP-T) foi desenvolvido por Frutuoso e Cruz (2006) para avaliar aspectos psicológicos em doenças crônicas relacionadas ao trabalho. A proposta desse instrumento é auxiliar na elaboração de diagnóstico, no planejamento do tratamento, além de ser útil no campo da ergonomia, já que fornece subsídios teóricos e metodológicos, para melhorar o ambiente e as condições de trabalho. A validade convergente do IP-T foi avaliada por meio das correlações entre os itens das dimensões: ansiedade, depressão e transtorno somatoforme do IAP-T e o Symptom Check List (SCL-90). Os índices de precisão indicaram alta correlação e validade convergente para as três dimensões (Frutuoso \& Cruz, 2006). Contudo, os autores 
afirmam que é importante dar continuidade à busca de validade convergente e de critério do instrumento.

A validade das inferências dos estudos realizados depende inteiramente da qualidade dos instrumentos de mensuração utilizados. Para tanto, é imprescindível submeter o instrumento à análise das propriedades psicométricas, para buscar evidências de fidedignidade e validade, para verificar se ele pode ser aplicado na população de estudo e avalia o construto para o qual foi desenvolvido (Alexandre \& Coluci, 2011).

A busca de evidências de validade para um novo instrumento deve avaliar sua covariância com outras medidas para as quais há evidências de validade disponíveis, o que é denominado de validade convergente. No caso do IP-T, trata-se da avaliação do grau com que o instrumento discrimina trabalhadores que diferem em determinada característica de acordo com o critério padrão ouro (Pasquali, 2013). A correlação do novo teste deve ser elevada, de pelo menos 0,75 para ser considerado válido. Além disso, o novo teste deve apresentar vantagens sobre o antigo, tais como tratar-se de um instrumento especifico para avaliar Transtorno Mental e Comportamental (TMC) relacionada ao trabalho, o qual ainda não foi validado para o Brasil.

Para avaliar a validade convergente do IP-T, foram utilizados o SQR-20 e a Escala de Ansiedade e Depressão (HADS). A escala foi originalmente desenvolvida para indicar a possível presença de estados de ansiedade e depressão em pacientes de ambulatório, não psiquiátricos, que estão com idade entre 16 e 64 anos (Zigmond \& Snaith, 1983). No Brasil, foram realizados estudos para comparar vantagens e desvantagens psicométricas entre a HADS, o Transtorno Geral de Ansiedade (GAD-7) e Questionário sobre Saúde do Paciente (PHQ-9) (Bergerot, Laros, \& Araújo, 2014). Os resultados do estudo indicaram que a escala HADS-D obteve as melhores características psicométricas: (1) coeficiente de fidedignidade de 0,84 ; (2) correlação média entre os itens de 0,42; (3) Erro Padrão da Medida (EPM) de 1,44; (4) carga fatorial média de 0,65; e (5) correlação item-total média de 0,58. A escala GAD-7 apresentou o segundo melhor resultado e a escala HADS-A apresentou: 1) coeficiente de fidedignidade de 0,74 ; (2) correlação média entre os itens de 0,28 ; (3) EPM de 1,63; (4) carga fatorial média de 0,53; e (5) correlação item-total média de 0,45 . O PHQ-9 obteve a menor qualidade psicométrica: (1) coeficiente de fidedignidade de 0,79 ; (2) correlação média entre os itens de 0,26; (3) EPM de 1,70; (4) carga fatorial média de 0,51; e (5) correlação item-total média de 0,45. Além disso,
Bergerot et al. (2014) indicaram que a escala HADS se mostrou adequada para avaliar a frequência de transtornos ansiedade e depressão em pacientes internados em hospitais. Contudo, ainda não foram realizados estudos com uma amostra de trabalhadores de indústrias de abate de suínos, aves e outros pequenos animais, e que estão afastados do trabalho por apresentar doença ocupacional, sendo necessário avaliar as propriedades psicométricas também nessa população.

Para que o instrumento seja considerado adequado para identificar transtornos mentais em uma determinada população - como, neste estudo, os trabalhadores de frigoríficos - é aconselhável conhecer não somente as características, mas, também, as propriedades psicométricas, tais como confiabilidade e diferente formas de validade, como a validade fatorial, validade convergente e discriminante. Dessa forma, o objetivo deste estudo foi avaliar as qualidades psicométricas do Inventário de Fatores Psicológicos em doenças relacionadas ao trabalho (IP-T), por meio da busca de evidências baseadas na estrutura interna, avaliada por análise fatorial exploratória, e evidências baseadas na relação com outras variáveis - em particular a correlação convergente entre ansiedade e depressão (HADS), transtornos leves (SQR-20) e fatores psicológicos relacionados ao trabalho (IP-T).

\section{Método}

\section{Participantes}

Participaram 353 trabalhadores da região oeste do Paraná, escolhidos por conveniência, que fizeram parte de dois grupos distintos: a) aqueles que estão exercendo suas atividades laborais $(63,2 \%, n=223)$; b) os afastados por apresentar doença ocupacional $(36,8 \%, n=$ 130). Dentre os trabalhadores afastados, $44,6 \%(n=58)$ são homens e 55,4\% $(n=72)$ são mulheres. Entre os que estavam trabalhando, $54,3 \%(n=121)$ são homens e $45,7 \%(n=102)$ são mulheres. Os homens afastados têm entre 19 e 79 anos $(M=46,3 ; D P=10,7)$, enquanto as mulheres têm entre 26 e 56 anos $(M=44,9 ; D P=$ $7,2)$. Dos trabalhadores, $57,2 \%(n=202)$ deles estavam empregados, na mesma empresa, há mais de cinco. A maioria $(88,8 \%, n=313)$ atuava na área de abate e de produção de produtos de carne e frequentou apenas o ensino fundamental $(47 \%, \mathrm{n}=166)$.

\section{Instrumentos}

O Inventário de Fatores Psicológicos em Doenças Relacionadas ao Trabalho - IP-T (Frutuoso \& 
Cruz, 2016) possui 103 itens agregados em cinco dimensões: na ansiedade (28 itens), são considerados os sintomas, como o medo e a ansiedade excessiva, que tendem a persistir por seis meses ou mais (DSM-V). Nos transtornos somatoformes (20 itens), são considerados os sintomas múltiplos e atuais que provocam sofrimento ou resultam em perturbação significativa da vida diária (DSM-V). Para caracterizar a dimensão depressão (20 itens), foram utilizados os critérios diagnósticos do Transtorno Depressivo Maior descritos no DSM-V. Na dimensão habilidades sociais (15 itens), foi considerado o conjunto de ações que levam o trabalhador a resolver problemas sociais com sucesso e, por fim, bem-estar no trabalho (20 itens), possui três características: subjetividade, predominância dos aspectos positivos e avaliação global favorável da vida. Há, na escala, 96 itens que são frases afirmativas, sendo 79 de correção positiva, 17 invertidas e sete itens são frases negativas, cuja correção é positiva, conforme a Tabela 1 apresenta.

As respostas são aferidas com base na frequência de ocorrência de cada item, em uma escala de 0 a 3 $(0=$ nunca, $1=$ às vezes, $2=$ geralmente, $3=$ sempre). Respostas com escores 0 e 1 representam a não ocorrência de sinais e sintomas indicadores de alteração psicológica, assim como os escores 2 e 3 são sinalizadores da sua ocorrência. $\mathrm{Na}$ correção positiva, foram somados os escores $(0,1,2,3)$ de todos os itens da dimensão, sendo a soma dividida pelo número de itens que a compõem (média aritmética simples para a soma dos escores obtidos). Na correção invertida, as respostas 0 e 1 foram alteradas respectivamente para 2 e 3 , e vice-versa, permitindo padronizar o tratamento estatístico dos dados para posteriormente determinar a média por dimensão analisada.
Self-Reporting Questionnaire (SRQ-20) (Organização Mundial de Saúde, 1994). Tem como objetivo avaliar a presença de transtorno mental e contém 20 itens dicotômicos, que são respondidas assinalando "sim" ou "não". O ponto de corte para diagnóstico de transtorno mental é a ocorrência de sete respostas positivas. O SQR-20 foi validado por Santos, Araújo, Pinho e Silva (2010), indicando sensibilidade e especificidade $(0,696$ a 0,882$)$ e satisfatória discriminação, embora tenha apresentado maior sensibilidade na avaliação de morbidade psíquica entre homens. Em geral, o SQR20 apresenta estudos nacionais e internacionais com desempenho aceitáveis no tocante à sensibilidade, especificidade e valores preditivos (Gonçalves et al., 2008; Ludemir \& Lewis, 2001).

Escala de Ansiedade e Depressão (HADS) (Zigmond \& Snaith, 1983). Utilizada na população geral, é composta por 14 itens, sendo sete deles (1, 3, $5,7,9,11$ e 13) voltados para avaliação da ansiedade (HADS-A) e sete $(2,4,6,8,10,12$ e 14), para a depressão (HADS-D). A subescala de ansiedade verifica a presença de sintomas de transtorno de ansiedade generalizada e a de depressão investiga, principalmente, a presença de anedonia, que se refere à perda da capacidade de cuidar das tarefas diárias de vida (Zigmond \& Snaith, 1983; Faro, 2015).

As respostas são de múltipla escolha e são aferidas com base na frequência de ocorrência de cada item. Para cada item, pode ser atribuído uma pontuação entre 0 e 3 , com 3 indicando frequência de sintomas mais elevados (Zigmond \& Snaith, 1983). As respostas dos itens que compõem as duas subescalas são somadas, para fornecer classificação separada para ansiedade e depressão. Na correção positiva, são somados os itens $2,4,7,9,12$ e 14, de acordo com a

Tabela 1

Quantidade de Itens com Frases Afirmativas e Negativa, e o Tipo de Correção

\begin{tabular}{llcc}
\hline Frase & Itens & $\begin{array}{c}\text { Correção } \\
\text { Total de } \\
\text { itens }\end{array}$ \\
\hline Afirmativa & $1,2,3,4,5,6,8,9,10,12,14,15,16,17,18,20,21,24,25,26,28,29,30$, & Positiva & 79 \\
& $31,32,33,35,36,37,39,40,41,42,43,44,45,47,48,49,50,51,53,54$, & & \\
& $56,57,58,59,60,61,62,63,64,65,68,69,70,72,73,74,75,76,77,78$, & & \\
& $80,81,83,85,86,87,88,90,92,93,95,96,97,98,99,100,101,103$ & & 17 \\
Afirmativa & $11,13,19,22,23,27,44,46,52,55,67,79,82,84,91,94,102$ & Invertida & 07 \\
Negativa & $7,34,38,39,66,71,89$ & Positiva & 07 \\
\hline
\end{tabular}

Fonte: Frutuoso \& Cruz, 2006. 
primeira alternativa de resposta, ou seja, no primeiro quadrante, zero para o menor valor e o último o maior valor, três. $\mathrm{Na}$ correção invertida, as respostas dos itens $1,3,5,6,8,10,11$ e 13 são alteradas respectivamente, nesse caso, a resposta do primeiro quadrante tem valor 3. A pontuação global em cada subescala vai de 0 a 21 , sendo que uma pontuação elevada corresponde a uma maior gravidade dos sintomas.

Botega, Bio, Zomignani, Garcia e Pereira (1995) obtiveram consistência interna de 0,68 e 0,77 nas subescalas de ansiedade e de depressão, respectivamente. A correlação dos itens com as respectivas subescalas sugere que elas possuem validades convergentes, não discriminantes. O ponto de corte indicativo de ansiedade igual ou maior que 8 e o escore indicativo de depressão igual ou superior a 9. A sensibilidade para HAD-A é de 93,7\% e para HAD-D 84,6\%. A especificidade para HAD-A é de 72,6\% e para HAD-D de 90,3\% (Botega et al., 1995). Os estudos realizados por Bergerot et al. (2014) corroboram com os resultados apresentados por Botega et al., (1995), indicando que a HADS se mostra adequada para avaliar a frequência de transtornos ansiedade e depressão em pacientes internados em hospitais no Brasil.

\section{Procedimentos de Coleta de Dados}

Os instrumentos foram aplicados no período entre 06/05/2015 a 30/08/2015, nos trabalhadores que estavam afastados, por apresentar doença ocupacional, e que foram aos sindicatos da alimentação nas três mesorregiões do oeste do Paraná (Cascavel, Toledo e Medianeira), para buscar auxílio médico ou jurídico. Além dos locais descritos, também foram realizadas coletas na Associação dos Portadores de LER/DORT (APLER) e o Sindicato dos Trabalhadores em Cooperativas Agrícolas de Medianeira. A coleta foi realizada em grupo, incialmente eram esclarecidos sobre o TCLE e, após, sobre as instruções de cada instrumento. Os que apresentavam dificuldades para escrever, por estar com alguma lesão, eram assessorados, ou seja, a pesquisadora lia cada item para obter as respostas verbais e após isso transcrevê-las para os questionários. O tempo médio utilizado por cada trabalhador foi de uma hora.

\section{Análise dos Dados}

Todos os dados obtidos por meio dos instrumentos foram processados no software Statistical Package for the Social Sciences (SPSS, versão 21). Para avaliar as propriedades psicométricas do IP-T, foram realizados três conjuntos de análise. A primeira, de avaliação da estrutura fatorial, baseou-se na análise das cargas fatoriais dos itens da escala nas cinco dimensões propostas a priori na definição do construto do instrumento. $\mathrm{O}$ modelo de análise fatorial exploratória foi ajustado pelo método de máxima verossimilhança e as cargas fatoriais consideradas foram ajustadas por meio da rotação ortogonal varimax. O segundo conjunto de análises buscou avaliar validade de critério, utilizando como critério concorrente se o trabalhador está afastado ou não. A predição do critério foi feita por meio de um conjunto de modelos de regressão logística simples com um único preditor cada: o escore nas dimensões dos instrumentos aplicados. O desempenho preditivo do IP-T e dos outros instrumentos aplicados foram avaliados em termos de acurácia, sensibilidade, especificidade e área sob a curva ROC (AUC-ROC) tanto para os dados de treinamento quanto por validação cruzada do tipo k-fold com dez partições.

O terceiro conjunto de análises avalia a validade de construto do IP-T por meio de uma análise fatorial dos diversos construtos mensurados pelos instrumentos empregados. Especial atenção foi dada à convergência e discriminação entre construtos similares mensurados por diferentes instrumentos. Por fim, foram avaliados os índices de fidedignidade das subescalas do IP-T, comparando-os com os outros instrumentos. A fidedignidade é apresentada utilizando dois descritores: $\lambda^{6}$ de Guttman e $\alpha$ de Cronbach.

\section{Consideraçôes Éticas}

O presente estudo seguiu as diretrizes propostas pelo Conselho Nacional de Saúde (CNS), na Resolução No 466 de 12 de dezembro de 2012, e foi aprovado pelo Comitê de Ética em Pesquisa com Seres Humanos da Plataforma Brasil sob o número de parecer 10488561.

\section{Resultados}

\section{Validade Fatorial}

Os cinco fatores do IP-T (ansiedade, transtornos somatoformes, depressão, habilidades sociais e bem-estar no trabalho) representam $41 \%$ da variância total explicada cumulativa. A análise da matriz de componente rotativa (varimax com normalização de Kaiser) foi estimada pressupondo os cinco fatores do instrumento. A Tabela 2 apresenta as dimensões do IP-T e seus respectivos itens vinculados por meio da análise fatorial exploratória.

Ao analisar o resultado da matriz dos fatores rotativa, pode-se observar que a maioria dos itens concentra 
Tabela 2

Analise Fatorial Exploratória do IP-T

\begin{tabular}{|c|c|c|c|c|c|c|c|c|}
\hline Item & $\mathrm{F} 1$ & $\mathrm{~F} 2$ & F3 & $\mathrm{F} 4$ & F5 & h2 & $\mathrm{u} 2$ & $\mathrm{com}$ \\
\hline IPT54 & 0.75 & & & & & 0.66 & 0.34 & 1.17 \\
\hline IPT56 & 0.75 & & & & & 0.61 & 0.39 & 1.07 \\
\hline IPT29 & 0.74 & & & & & 0.56 & 0.44 & 1.03 \\
\hline IPT36 & 0.72 & & & & & 0.45 & 0.55 & 1.12 \\
\hline IPT43 & 0.68 & & & & & 0.61 & 0.39 & 1.08 \\
\hline IPT49 & 0.65 & & & & & 0.74 & 0.26 & 1.29 \\
\hline IPT66 & 0.62 & & & & & 0.36 & 0.64 & 1.03 \\
\hline IPT10 & 0.61 & & & & & 0.53 & 0.47 & 1.27 \\
\hline IPT31 & 0.61 & & & & & 0.47 & 0.53 & 1.06 \\
\hline IPT25 & 0.57 & & & & & 0.40 & 0.60 & 1.09 \\
\hline IPT14 & 0.56 & & & & & 0.38 & 0.62 & 1.28 \\
\hline IPT21 & 0.54 & & & & & 0.45 & 0.55 & 1.30 \\
\hline IPT16 & 0.54 & & & & & 0.50 & 0.50 & 1.26 \\
\hline IPT69 & 0.54 & & & & & 0.42 & 0.58 & 1.21 \\
\hline IPT45 & 0.54 & & & & & 0.45 & 0.55 & 1.24 \\
\hline IPT12 & 0.53 & & & & & 0.44 & 0.56 & 1.22 \\
\hline IPT32 & 0.52 & & & & & 0.49 & 0.51 & 1.49 \\
\hline IPT74 & 0.51 & & & & & 0.55 & 0.45 & 1.58 \\
\hline IPT61 & 0.51 & & & 0.31 & & 0.69 & 0.31 & 1.93 \\
\hline IPT41 & 0.51 & & & & & 0.47 & 0.53 & 1.59 \\
\hline IPT15 & 0.50 & & & & & 0.61 & 0.39 & 1.65 \\
\hline IPT77 & 0.49 & & & & & 0.37 & 0.63 & 1.16 \\
\hline IPT85 & 0.48 & & & & & 0.42 & 0.58 & 1.48 \\
\hline IPT47 & 0.47 & & & & & 0.39 & 0.61 & 1.34 \\
\hline IPT75 & 0.47 & & & & & 0.51 & 0.49 & 1.67 \\
\hline IPT4 & 0.44 & & & & & 0.23 & 0.77 & 1.16 \\
\hline IPT50 & 0.43 & & & & & 0.33 & 0.67 & 1.47 \\
\hline IPT17 & 0.43 & 0.33 & & & & 0.45 & 0.55 & 2.38 \\
\hline IPT30 & 0.43 & & & & & 0.32 & 0.68 & 1.36 \\
\hline IPT58 & 0.42 & & & & & 0.52 & 0.48 & 2.08 \\
\hline IPT6 & 0.40 & 0.34 & & & & 0.50 & 0.50 & 2.38 \\
\hline IPT70 & 0.39 & & & & & 0.36 & 0.64 & 1.80 \\
\hline IPT78 & 0.37 & & & & & 0.54 & 0.46 & 2.64 \\
\hline IPT57 & 0.37 & & & & & 0.50 & 0.50 & 2.62 \\
\hline IPT3 & 0.36 & & & & & 0.24 & 0.76 & 1.28 \\
\hline IPT18 & 0.36 & & & & & 0.42 & 0.58 & 2.22 \\
\hline IPT52 & -0.36 & & & & -0.34 & 0.32 & 0.68 & 2.05 \\
\hline IPT24 & 0.35 & & & & & 0.26 & 0.74 & 2.19 \\
\hline IPT9 & 0.35 & & & & & 0.31 & 0.69 & 2.11 \\
\hline IPT20 & 0.34 & & & & & 0.25 & 0.75 & 2.00 \\
\hline
\end{tabular}


Tabela 2

Analise Fatorial Exploratória do IP-T (Continuação)

\begin{tabular}{|c|c|c|c|c|c|c|c|c|}
\hline Item & F1 & $\mathrm{F} 2$ & F3 & $\mathrm{F} 4$ & F5 & h2 & $\mathrm{u} 2$ & com \\
\hline IPT2 & & & & & & 0.19 & 0.81 & 1.56 \\
\hline IPT42 & & & & & & 0.41 & 0.59 & 2.87 \\
\hline IPT1 & & & & & & 0.18 & 0.82 & 2.80 \\
\hline IPT59 & & & & & & 0.24 & 0.76 & 2.58 \\
\hline IPT26 & & & & & & 0.13 & 0.87 & 2.65 \\
\hline IPT81 & & & & & & 0.25 & 0.75 & 2.34 \\
\hline IPT68 & & & & & & 0.30 & 0.70 & 3.46 \\
\hline IPT8 & & & & & & 0.12 & 0.88 & 1.97 \\
\hline IPT53 & & 0.90 & & & & 0.81 & 0.19 & 1.01 \\
\hline IPT96 & & 0.81 & & & & 0.70 & 0.30 & 1.27 \\
\hline IPT33 & & 0.79 & & & & 0.67 & 0.33 & 1.03 \\
\hline IPT63 & & 0.73 & & & & 0.60 & 0.40 & 1.03 \\
\hline IPT48 & & 0.70 & & & & 0.62 & 0.38 & 1.24 \\
\hline IPT60 & & 0.68 & & & & 0.59 & 0.41 & 1.06 \\
\hline IPT40 & & 0.60 & & & & 0.40 & 0.60 & 1.08 \\
\hline IPT34 & & 0.59 & & & & 0.50 & 0.50 & 1.59 \\
\hline IPT92 & & 0.57 & & & & 0.55 & 0.45 & 1.25 \\
\hline IPT83 & & 0.56 & & & & 0.50 & 0.50 & 1.47 \\
\hline IPT97 & & 0.55 & & & & 0.57 & 0.43 & 1.49 \\
\hline IPT5 & & 0.51 & & & & 0.42 & 0.58 & 1.24 \\
\hline IPT35 & & 0.50 & & & & 0.48 & 0.52 & 1.52 \\
\hline IPT7 & & 0.38 & & & & 0.19 & 0.81 & 1.73 \\
\hline IPT73 & & 0.30 & & & & 0.44 & 0.56 & 3.42 \\
\hline IPT79 & & & & & & 0.42 & 0.58 & 3.49 \\
\hline IPT27 & & & 0.57 & & & 0.43 & 0.57 & 1.78 \\
\hline IPT13 & & & 0.54 & & & 0.28 & 0.72 & 1.10 \\
\hline IPT23 & & & 0.53 & & & 0.51 & 0.49 & 1.74 \\
\hline IPT22 & & & 0.51 & & & 0.27 & 0.73 & 1.11 \\
\hline IPT55 & & & 0.48 & & & 0.23 & 0.77 & 1.21 \\
\hline IPT19 & & & 0.47 & & & 0.25 & 0.75 & 1.46 \\
\hline IPT44 & & -0.33 & 0.43 & & & 0.28 & 0.72 & 2.48 \\
\hline IPT102 & & & 0.43 & & & 0.26 & 0.74 & 1.88 \\
\hline IPT11 & & & 0.41 & & & 0.17 & 0.83 & 1.15 \\
\hline IPT94 & & & 0.41 & & & 0.17 & 0.83 & 1.26 \\
\hline IPT82 & & & 0.41 & & & 0.22 & 0.78 & 1.81 \\
\hline IPT46 & & & 0.40 & & & 0.23 & 0.77 & 2.42 \\
\hline IPT67 & & & 0.33 & & & 0.16 & 0.84 & 3.00 \\
\hline IPT84 & & 0.32 & 0.33 & & & 0.26 & 0.74 & 2.02 \\
\hline IPT91 & & & 0.32 & & & 0.12 & 0.88 & 2.06 \\
\hline IPT76 & & & 0.32 & & & 0.17 & 0.83 & 2.01 \\
\hline
\end{tabular}


Tabela 2

Analise Fatorial Exploratória do IP-T (Continuação)

\begin{tabular}{|c|c|c|c|c|c|c|c|c|}
\hline Item & $\mathrm{F} 1$ & F2 & $\mathrm{F} 3$ & $\mathrm{~F} 4$ & F5 & h2 & $\mathrm{u} 2$ & com \\
\hline IPT93 & & & & 0.56 & & 0.50 & 0.50 & 1.20 \\
\hline IPT101 & & & & 0.54 & & 0.45 & 0.55 & 1.35 \\
\hline IPT103 & & & & 0.52 & & 0.43 & 0.57 & 1.37 \\
\hline IPT98 & & & & 0.43 & & 0.21 & 0.79 & 1.07 \\
\hline IPT90 & & & & 0.43 & & 0.40 & 0.60 & 1.82 \\
\hline IPT100 & & & & 0.42 & & 0.46 & 0.54 & 1.91 \\
\hline IPT95 & 0.39 & & & 0.40 & & 0.56 & 0.44 & 2.58 \\
\hline IPT86 & 0.36 & & & 0.40 & & 0.50 & 0.50 & 2.20 \\
\hline IPT72 & & & & 0.36 & & 0.34 & 0.66 & 2.15 \\
\hline IPT88 & & & & 0.34 & & 0.30 & 0.70 & 2.51 \\
\hline IPT99 & & & & 0.43 & 0.45 & 0.49 & 0.51 & 2.11 \\
\hline IPT37 & & & & & 0.43 & 0.37 & 0.63 & 1.65 \\
\hline IPT80 & & & & & 0.37 & 0.41 & 0.59 & 2.33 \\
\hline IPT39 & & 0.32 & & & 0.37 & 0.31 & 0.69 & 1.97 \\
\hline IPT28 & & & & & 0.36 & 0.29 & 0.71 & 1.81 \\
\hline IPT38 & & & & & 0.34 & 0.30 & 0.70 & 2.12 \\
\hline IPT64 & & 0.31 & & & 0.32 & 0.53 & 0.47 & 3.20 \\
\hline IPT51 & & & & & 0.32 & 0.37 & 0.63 & 2.77 \\
\hline IPT87 & & & & & & 0.15 & 0.85 & 2.15 \\
\hline IPT71 & & & & & & 0.22 & 0.78 & 2.60 \\
\hline IPT62 & & & & & & 0.32 & 0.68 & 2.96 \\
\hline IPT65 & & & & & & 0.20 & 0.80 & 3.39 \\
\hline IPT89 & & & & & & 0.21 & 0.79 & 2.57 \\
\hline
\end{tabular}

maior força no primeiro fator. Esse resultado indica que apenas um fator responde por $35,5 \%$ da variância total explicada cumulativa. Somado a isso, muitos itens estão representados em mais de um fator, como, por exemplo, o número 14 (Irrito-me facilmente com as pessoas) que tem praticamente a mesma carga no fator $1(0,444)$ e no fator $3(0,447)$. Além disso, os itens 23 , 26, 70, 76 e 87 mostraram não ser representativos de nenhuma das dimensões. Esse resultado aponta que é necessário rever os itens, já que podem estar gerando viés de confusão. Para tanto, todos esses itens foram retirados do inventário, além daqueles que apresentaram carga máxima inferior a 0,30 , resultando em uma redução de 53 itens.

Os itens também carregaram em fatores diferentes do esperado. Ansiedade e depressão carregam principalmente no fator 1 , o que gera dificuldade para discriminar entre as duas dimensões na comparação com outras escalas. Já o fator 2 refere-se principalmente aos itens do transtorno somatoforme, mas nessa dimensão contém ainda vários itens relativos ao fator 1, de ansiedade e depressão, o que aponta a necessidade de também analisar e avaliar o conteúdo destes itens. A dimensão bem-estar no trabalho está razoavelmente coerente, com a maioria dos itens carregando no fator 4 e um único item carregou no fator 2 . Além disso, todos os itens que carregaram no fator 4 possuem carga negativa. Ao comparar com a primeira versão do IP-T, pode-se observar que esses itens são de correção invertida.

A dimensão habilidade social precisa ser melhor compreendida em função dos itens carregarem em fatores diferentes. Além disso, o fator 5 carregou apenas itens que pesaram em outros fatores. Esse 
resultado indica que, além dos itens, faz-se necessário também rever as dimensões que compõem a escala. As dimensões bem-estar e ansiedade foram as que apresentaram melhor discriminação de itens na análise fatorial.

\section{Validade de Critério do IP-T}

Para analisar quão bem o IP-T permite discriminar entre trabalhadores afastados (com problemas de saúde) e trabalhando normalmente (a princípio, sem problemas de saúde), foram ajustados vários modelos de regressão logística. Em todos os modelos, a variável de desfecho é a condição do trabalhador: afastado (1) ou trabalhando normalmente (0). Cada modelo foi construído com apenas uma variável preditiva, o escore do sujeito nas escalas aplicadas. Para facilitar a comparação entre os diferentes preditores, os valores foram padronizados como escores-z. Foram também testados os modelos que incluíam uma variável indicadora do sexo do participante e sua interação com o preditor principal, mas como o acréscimo dessa informação não melhorou significativamente o poder preditivo dos modelos. A Tabela 3 apresenta os coeficientes obtidos em cada regressão logística, a transformação desses coeficientes em razão de chances e a área sob a curva ROC (AUC-ROC), em conjunto com os erros-padrões de cada estimativa em parênteses.

Todas as variáveis consideradas são preditoras positivas da condição de afastamento. As razões de chances de cada variável são elevadas, chegando a 4,4 para cada desvio padrão na escala de Transtorno Somatoforme do IP-T. O escore de Habilidades Sociais e de Bem-Estar no Trabalho, ambos do IP-T, também predizem positivamente a condição de afastamento, ainda que seus coeficientes sejam menores.

A área sob a curva (AUC) da ROC de todos os modelos é maior que $50 \%$, indicando sua adequação em predizer os casos de afastamento. Convém lembrar, porém, que os dados de ajuste e de predição são os mesmos, o que significa que as estimativas estão superestimando a qualidade preditora das variáveis. A maioria das AUC está entre $73 \%$ e $78 \%$, com exceção do Transtorno Somatoforme e Habilidades Sociais, que estão nos extremos dos valores com $83 \%$ e $64 \%$, respectivamente.

Apesar do desempenho mediano das escalas de Habilidades Sociais e Bem-Estar no Trabalho do IP-T, as outras subescalas do instrumento avaliado no presente artigo tem desempenho preditivo comparável a outros instrumentos (Frutuoso \& Cruz, 2006). Em particular, os escores de Transtorno Somatoforme do IP-T apresentam desempenho preditivo superior a qualquer outra medida considerada neste estudo.

Entretanto, o interesse é verificar em que medida a escala IP-T atinge a situação-alvo, ou seja, o afastamento de trabalhadores por questões de saúde. Neste quesito, denota-se que algumas subescalas do IP-T apresentam desempenhos satisfatórios e até melhores que os instrumentos comparados, com base nos critérios escolhidos, indicativo de sua validade com relação aos fenômenos que se propõe mensurar.

\section{Validade Convergente e Discriminante do IP-T}

Foram verificadas as validades convergente $\mathrm{e}$ discriminante das subescalas do IP-T, entre si e com os outros instrumentos aplicados. Por se tratar da

Tabela 3

Comparação dos Diferentes Modelos de Regressão Logística para Predição da Condição do Trabalhador em Função do Escore da Variável Indicada

\begin{tabular}{lccc}
\hline Variável preditora & $\begin{array}{c}\text { Coeficiente } \\
(\mathrm{EP})\end{array}$ & $\begin{array}{c}\text { Razão de Chances } \\
(\mathrm{EP})\end{array}$ & $\begin{array}{c}\text { Área sob ROC } \\
(\mathrm{EP})\end{array}$ \\
\hline HADSD & $1(0,13)$ & $2,8(1,03)$ & $0,77(0,03)$ \\
HADSA & $1,1(0,14)$ & $3,1(1,18)$ & $0,78(0,03)$ \\
SQR20 & $1,2(0,14)$ & $3,3(1,24)$ & $0,8(0,03)$ \\
IPT-Ansiedade & $0,84(0,13)$ & $2,3(0,82)$ & $0,73(0,03)$ \\
IPT-Trans. Somato. & $1,5(0,16)$ & $4,4(1,79)$ & $0,84(0,022)$ \\
IPT-Depressão & $0,94(0,13)$ & $2,6(0,93)$ & $0,75(0,03)$ \\
IPT-Hab. Soc. & $0,53(0,12)$ & $1,7(0,58)$ & $0,64(0,03)$ \\
IPT-Trabalho & $0,96(0,14)$ & $2,6(0,97)$ & $0,75(0,03)$ \\
\hline
\end{tabular}


comparação dos escores totais de cada escala, utilizamos a correlação de Pearson, uma vez que as variáveis em questão são praticamente contínuas. Foram analisadas as correlações entre as variáveis latentes mensuradas por cada instrumento, assim como a matriz de correlação desatenuada, utilizando o índice de $\alpha$ de Cronbach como medida de fidedignidade para corrigir a correlação entre os escores (Tabela 4).

A matriz de correlação entre as escalas utilizadas na pesquisa é bastante uniforme. A maioria das correlações se encontra entre 0,80 e 0,91 (implicando, portanto de $64 \%$ a $82 \%$ da variância compartilhada). As correlações mais fortes acontecem entre as três primeiras subescalas do IP-T, o HADS e o SQR20. As subescalas de Habilidades Sociais e de Bem-estar no Trabalho também estão positivamente correlacionadas às outras variáveis, mas com menos força. Mesmo assim, seus coeficientes de correlação variam de 0,70 a 0,87 , índices consideravelmente altos.

A homogeneidade das correlações sugere que há apenas um fator comum responsável pela maior parte da variância das diferentes escalas. Em outras palavras, há forte convergência das escalas entre si. As subescalas de Habilidades Sociais e de Bem-estar no trabalho poderiam compor outras duas dimensões separadas, mas a força de suas correlações com as outras variáveis não justificaria, a princípio, um ou mais fatores distintos do principal. Esse resultado, em conjunto com a alta correlação entre escalas que se propõe mensurar ansiedade e depressão indicam pouca discriminação entre os construtos, novamente apontando para a solução de um único fator. Para averiguar se a solução de um único fator é de fato a melhor, foi realizada à análise paralela de Horn baseada na extração de componentes principais de bases de dados aleatórias. A análise paralela, em conjunto com o scree-plot, ajudam a diagnosticar visualmente a melhor solução.

Tanto utilizando o critério de Kaiser (eigenvalues acima de 1), quanto localizando o ponto no qual há uma forte desaceleração na diminuição dos valores próprios, ou então pelas simulações de dados aleatórios da análise paralela, a solução de um único fator parece ser a mais indicada. O critério Mínima Média Parcial (Minimum Average Partial) de Velicer também é minimizado com um único fator.

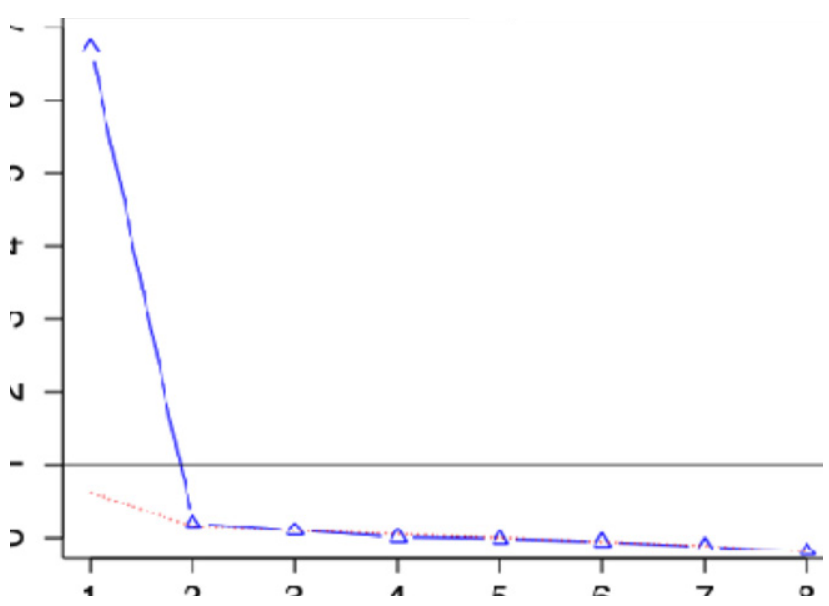

Figura 1. Análise paralela e scree-plot das escalas.

Tabela 4

Matriz de Correlação Desatenuada dos Índices dos Instrumentos Comparados

\begin{tabular}{lcccccccc}
\hline & \multirow{2}{*}{} & \multirow{2}{*}{ QRS-20 } & HADSA & \multirow{2}{*}{ HADSD } & \multicolumn{5}{c}{ IP-T* } \\
\cline { 5 - 9 } & & & & Ans & TSom & Dep & HSoc & BEsTr \\
\hline QRS-20 & 1 & 0,89 & 0,92 & 0,88 & 0,89 & 0,91 & 0,76 & 0,76 \\
HADSA & 0,89 & 1 & 0,91 & 0,88 & 0,87 & 0,86 & 0,72 & 0,74 \\
HADSD & 0,92 & 0,91 & 1 & 0,87 & 0,81 & 0,92 & 0,79 & 0,81 \\
IP-T Ans & 0,88 & 0,88 & 0,87 & 1 & 0,89 & 0,96 & 0,87 & 0,73 \\
IP-T-TSom & 0,89 & 0,87 & 0,81 & 0,89 & 1 & 0,9 & 0,7 & 0,75 \\
IP-T-Dep & 0,91 & 0,86 & 0,92 & 0,96 & 0,9 & 1 & 0,88 & 0,81 \\
IP-T-HSoc & 0,76 & 0,72 & 0,79 & 0,87 & 0,7 & 0,88 & 1 & 0,82 \\
IP-T-BEsTr & 0,76 & 0,74 & 0,81 & 0,73 & 0,75 & 0,81 & 0,82 & 1 \\
\hline
\end{tabular}

Nota. O IP-T possui 5 dimensões que são: ansiedade (Ans), transtorno Somatoforme (TSom), depressão (Dep), habilidades sociais (HSoc) e bem estar no trabalho (BEsTr). 
Apesar de alguns dos critérios indicarem outras soluções possíveis, é evidente que, se for considerada a parcimônia do modelo, a solução de um fator parece ser suficiente para explicar a maior parte da variação entre as diferentes escalas e subescalas. Com base nessa escolha, foi realizada a análise fatorial das medidas para identificar sua fidedignidade com o fator latente. Sendo uma solução de fator único, não foi feita nenhuma rotação da matriz de cargas.

As cargas das escalas e subescalas foram consideravelmente altas, indo de 0,82 para os escores de Habilidades Sociais e Bem-estar no Trabalho até 0,98 para Depressão e Ansiedade do IP-T. As comunalidades $\left(b^{2}\right)$ podem ser interpretadas aqui como índices de fidedignidade $\left(r_{x x}\right)$ das diferentes escalas em relação a sua determinação por um único fator latente. A maioria das comunalidades é consideravelmente alta, entre 0,68 e 0,97 , ou seja, $68 \%$ a $97 \%$ da variação dos escores pode ser atribuída à variável latente de interesse. Isso indica a forte convergência entre a maioria das escalas utilizadas.

Essa forte determinação por um único fator, apesar de referendar a validade convergente do IP-T considerando as outras escalas utilizadas, também indica uma fraca discriminação entre construtos. É esperado que os construtos considerados pelos instrumentos, como ansiedade, depressão, habilidades sociais, sejam correlacionados; os dados, porém, indicam que essa correlação pode ser atribuída a um único fator latente, o que invalida a teoria de que há construtos distintos. Esse problema não afeta apenas o IP-T, mas também o HADS e o SRQ20.

As subescalas do IP-T para avaliar Habilidades Sociais e Bem-estar no Trabalho também estão fortemente correlacionadas a este mesmo fator, mas sua variância residual é um pouco mais alta. Isso pode ser interpretado como um fraco indício de discriminação dessas duas subescalas com relação às outras. Porém, é necessário levar em consideração que não há discriminação clara entre os mesmos construtos mensurados por diferentes instrumentos.

\section{Análise da Fidedignidade das Escalas}

Para finalizar a análise das propriedades psicométricas do IP-T, a fidedignidade de suas subescalas foram computadas e comparadas às fidedignidades dos outros instrumentos aplicados. Foi utilizado tanto o $\alpha$ de Cronbach quanto o $\lambda_{6}$ de Guttman. O índice $\lambda_{6}$ é mais robusto no caso de itens heterogêneos, com grande variação nas cargas fatoriais; por outro lado, o $\alpha$ estima o limite inferior de fidedignidade com maior precisão no caso de itens homogêneos. Por outro lado, como cada escala é composta apenas por itens que se supõe estão relacionados exclusivamente com a variável latente de interesse, o $\alpha$ de Cronbach e o $\lambda_{6}$ de Guttman produzem estimativas semelhantes do limite inferior $\mathrm{da}$ fidedignidade.

Os índices de fidedignidade estão dentro do limite aceitável. O maior índice, para o IP-T Ansiedade, é de 0,94 (0,95); o menor índice, também do IP-T, é da subescala de Habilidades Sociais: 0,74 (0,78). Esse valor pode ser parcialmente explicado pelo fato de que o item 52 correlacionou-se negativamente com a escala e deveria ser invertido. Porém, manteve-se o padrão de correção original da escala. Os índices de fidedignidade intraescalas concordam parcialmente com os índices de fidedignidade interescalas encontrado pela análise fatorial dos escores. De maneira geral, os escores das escalas de ansiedade, transtornos somatoformes e depressão são tão confiáveis quanto às escalas utilizadas para comparação.

Tabela 5

Análise Fatorial das Escalas Considerando Solução de um Único Fator

\begin{tabular}{lccc}
\hline Escalas & Carga Fatorial & $\mathrm{h}^{2}$ & $\mathrm{u}^{2}$ \\
\hline IP-T Depressão & 0,98 & 0,97 & 0,034 \\
IP-T Ansiedade & 0,97 & 0,93 & 0,068 \\
HADSD & 0,94 & 0,89 & 0,113 \\
SRQ20 & 0,94 & 0,88 & 0,12 \\
HADSA & 0,92 & 0,85 & 0,154 \\
IP-T T.S. & 0,91 & 0,84 & 0,165 \\
IP-T Habilidades Sociais & 0,87 & 0,76 & 0,24 \\
IP-T Bem-Estar no Trabalho & 0,82 & 0,68 & 0,32 \\
\hline
\end{tabular}




\section{Discussão}

Este estudo avaliou as propriedades psicométricas do IP-T. A amostra foi de 355 trabalhadores da região oeste do Paraná, os quais estão divididos em dois grupos: os trabalhadores afastados $(36,3 \%)$ por apresentar doença ocupacional e os que não estão afastados (62,3\%), (considerados com saúde). Em amostras de pessoas com dor e sem dor, as dimensões Ansiedade, Depressão e Transtorno Somatoforme da IP-T apresentaram maior consistência interna (Frutuoso \& Cruz, 2006). Neste estudo, também foi analisado quão bem o IP-T permite discriminar entre trabalhadores afastados (com problemas de saúde) e trabalhando não afastados. A curva ROC ficou entre 0,64 e 0,84 , indicando que todas as subescalas do IP-T se mostraram preditores positivas da condição de afastamento por doença ocupacional.

Frutuoso e Cruz (2006) analisaram, ainda, a consistência externa das dimensões, ansiedade, depressão e transtorno somatoforme do SCL-90-R, que é um inventário desenvolvido para identificar e avaliar sintomas psicológicos, com as mesmas dimensões do IP-T. Os resultados indicaram alta correlação e consistência externa, o que sugere que os itens das dimensões descritas, dos dois instrumentos, medem propriedades psicológicas similares.

No presente estudo, foram utilizados três instrumentos, ao comparar a habilidade das subescalas do IP-T em predizer a situação de afastamento do trabalho com as outras escalas utilizadas, ficou evidente que o IP-T consegue discriminar os casos com acurácia semelhante e também superior à das outras escalas. Nesse âmbito, considerando o critério de diagnosticar a situação de um trabalhador, há evidência de validade de critério do IP-T.

Os padrões de correlação entre os escores das escalas sugere uma forte convergência entre elas. A análise fatorial exploratória indica que a solução unifatorial é, de fato, a melhor para representar a variância compartilhada entre as variáveis. Isso indica que há evidência para a validade convergente das subescalas do IP-T com as outras escalas utilizadas para comparação.

Essa convergência, porém, traz um problema preocupante: há pouca evidência de discriminação entre as diferentes dimensões dos construtos utilizados. A solução unifatorial parece implicar a existência de um único fator comum a todas as escalas e subescalas, mas os construtos teóricos pressupõem a existência de mais fatores: o HADS deveria ter um fator relativo à ansiedade e outro à depressão, mas não há diferença entre eles nos dados; da mesma forma, o IP-T possui cinco dimensões, mas apenas o Bem-Estar no Trabalho e Habilidades Sociais parecem se destacar - e muito fracamente - da influência de um único fator.

Ao analisar cada dimensão em particular, denota-se que as dimensões: habilidades sociais, transtorno somatoforme e bem-estar no trabalho, os itens de cada uma destas carregam em sua maioria no mesmo fator, mas ainda precisam sem melhor discriminados. Como exemplo, na dimensão bem-estar no trabalho, em sua maioria, os itens carregam no fator 4; ao analisar cada item individualmente denota-se que eles realmente referem-se à categoria "trabalho", com exceção do item número 5 (Ao executar as atividades de trabalho sinto muito cansaço), que está na mesma dimensão, porém carrega no fator 2 (representa momentaneamente o Transtorno Somatoforme). Nesse caso, é possível verificar que na análise fatorial a importância

Tabela 6

Análise de Fidedignidade das Escalas

\begin{tabular}{lcc}
\hline Escalas & Alfa de Cronbach $(\alpha)$ & Guttman $\left(\lambda_{\alpha}\right)$ \\
\hline SRQ20 & 0,92 & 0,93 \\
HADSA & 0,88 & 0,87 \\
HADSD & 0,84 & 0,83 \\
IP-T Ansiedade & 0,94 & 0,95 \\
IP-T Transtornos Somatoformes & 0,93 & 0,94 \\
IP-T Depressão & 0,91 & 0,93 \\
IP-T Habilidades Sociais & 0,74 & 0,78 \\
IP-T Bem-Estar no Trabalho & 0,82 & 0,84 \\
\hline
\end{tabular}


foi dada ao tema "cansaço" e não para o "trabalho", dando ênfase assim ao sentimento negativo. É aconselhado, então, rever o conteúdo deste item, bem como todos os das demais dimensões, que carregam em fator diferente, para melhor atender a dimensão ou transferi-lo para o fator correspondente, no caso do exemplo citado, o fator 2.

Por meio dos sintomas mais evidentes descritos para nas dimensões ansiedade e depressão, ao analisar os itens do transtorno depressivo, de acordo com o DSM-V, eles traduzem bem as características melancólicas referentes a este transtorno. Da mesma forma, o item da dimensão ansiedade atende a característica de que o medo é um sentimento apresentado por pessoas que apresentam transtorno ansioso. Se os itens construídos estão de acordo com os principais sintomas descritos para cada transtorno, então como explicar o resultado da análise fatorial que carrega o fator 1 tanto para ansiedade quanto para depressão?

Nas patologias de ansiedade, os ataques de pânico se destacam como um tipo particular de resposta ao medo, porém não estão limitados aos transtornos de ansiedade e também podem ser vistos em outros transtornos mentais (Borsonello, Santos, Schmidt \& Andrade, 2002). Assim, os transtornos depressivos podem ter além da caraterística melancólicas outros atributos mistos associados a características ansiosas como, por exemplo, agitação, insônia, fuga de ideias ou experiência subjetiva de que os pensamentos estão acelerados. Todos esses sintomas, apesar de representar o transtorno depressivo, podem ser facilmente confundidos com o transtorno ansioso.

Como os itens das dimensões ansiedade e depressão carregam no mesmo fator 1 , mas deveriam representar sintomas dos dois transtornos, para aumentar a distância entre as duas dimensões sugere-se que seja realizada uma escolha entre um dos diagnósticos de transtorno depressivo, apresentados no DSM-V e um entre os diagnósticos de transtornos ansiosos. Neste caso, para melhor descrever cada dimensão de forma mais clara, os itens devem ser revistos para atender a dimensão para cada qual está representado.

Entre os diagnósticos apresentados no Manual de Diagnóstico e Estatística dos Transtornos Mentais, quinta edição - DSM-V - os sintomas descritos no transtorno de ansiedade generalizada (F41.1) e no transtorno depressivo maior moderado (F32.1) possuem critérios diagnósticos bem definidos, a tal ponto de se diferenciar um do outro (DSM-V, 2013). Com base nos critérios diagnósticos de cada dimensão, denota-se que se forem alterados somente os itens da dimensão ansiedade, para corresponder mais a descrição de um transtorno de ansiedade generalizada pode haver uma distinção mais precisa entre este e o transtorno depressivo.

Os índices de fidedignidade das escalas estão dentro do aceitável. Porém, esses índices foram computados utilizando a chave original das escalas. Não foi verificado se os itens permaneceram estáveis em suas cargas nos seus respectivos fatores. Assim, sugere-se que novos estudos sejam realizados, para que seja novamente analisado se as cargas dos itens permanecem estáveis nos seus referentes fatores.

A amostra de conveniência para a realização das análises não permite generalizar os achados sobre $\mathrm{O}$ IP-T para populações diversas da estudada, limitando o alcance das conclusões sobre as propriedades psicométricas do instrumento. A análise fatorial dos itens da escala sugere a necessidade de se readequar a dimensionalidade do constructo e, principalmente, a avaliação da pertinência dos itens, exigindo novas pesquisas para a seleção dos itens mais representativos das dimensões e uma possível re-especificação do número de fatores latentes. O desempenho preditivo das subescalas do IP-T foi estimado a partir da mesma amostra utilizada para o treinamento dos modelos logísticos e, por isso, devem estar superestimando a capacidade dos instrumentos em discriminar trabalhadores doentes e saudáveis. Estudos com trabalhadores de diferentes contextos são necessário para que a habilidade diagnóstica do IP-T possa ser referendada e generalizada.

\section{Referências}

Alexandre, N. M., \& Coluci, M. C. (2011). Validade de conteúdo nos processos de construção e adaptação de instrumentos de medidas. Ciência e Saúde Coletiva. Rio de Janeiro, 16(7), 3061-3068. doi: 10.1590/ S1413-81232011000800006

American Psychiatry Association. (2013). Diagnostic and Statistical Manual of MentalDisorders - DSM-5. 5th. ed. Washington, DC: Author.

Bergerot,C.D.,Laros,J.A.,\&Araújo,T.C.(2014).Avaliação de ansiedade e depressão em pacientes oncológicos: Comparação psicométrica. Psico-USF, 19(2), 187-197. doi: 10.1590/1413-82712014019002004.

Blacker, D., \& Endicott, J. (2002). Psychometric properties: Concepts of reliability and validity. Handbook 
of Psychiatric Measures. Washingtons: American Psychiatric Association, 7-14.

Bolsoni, L. M., \& Zuardi, A. W. (2015). Estudos psicométricos de instrumentos breves de rastreio para múltiplos transtornos mentais. Jornal Brasileiro de Psiquiatria, 64(1), 63-69. doi:10.1590/0047-2085000000058

Borsonello, E. C., Santos, L. C., Schmidt, M. L. G., \& Andrade, T. G. C. S. (2002). A influência do afastamento por acidente de trabalho sobre a ocorrência de transtornos psíquicos e somáticos. Psicologia: Ciência e Profissão, 22(3), 32-37. doi: 10.1590/S1414-98932002000300006

Botega, N. J., Bio, M. R., Zomignani, M. A., Garcia Jr, C., \& Pereira, W. A. B. (1995). Transtornos do humor em enfermaria de clínica médica e validação de escala de medida (HAD) de ansiedade e depressão. Revista de Saúde Pública, 29(5), 355-363. doi: 10.1590/S0034-89101995000500004

Cronbach L. J. (1951). Coefficient alpha and the internal structure of tests. Psychometrika, 16(3), 297-334. doi:10.1007/BF02310555

Conselho Nacional de Saúde. (2012). Resolução n 466, de 12 de dezembro de 2012. Brasilia, DF: Author. Recuperado de http://www.conselho.saude.gov.br/ web_comissoes/conep/index.html

Cruz, R. M. (2010). Nexo técnico e vigilância à saúde do trabalhador: Uma agenda científica para o NTEP. Em J. Machado, L. Soratto \& W. Codo (Eds.), Saúde e trabalho no Brasil: uma revolução silenciosa (pp. 256272). Petrópolis: Vozes.

Erthal, T. C. (2011). Manual de Psicometria. 6a ed. Rio de Janeiro: Jorge Zahar.

Faro, A. (2015). Análise Fatorial Confirmatória e Normatização da Hospital Anxiety and Depression Scale (HADS). Psicologia: Teoria e Pesquisa. 31(3), 349353. doi: 10.1590/0102-37722015032072349353.

Frutuoso, J. T., \& Cruz, R. M. (2006). Mensuração de aspectos psicológicos presentes em portadores de dor crônica relacionada ao trabalho (Tese de doutorado). Programa de Pós-graduação da Engenharia de Produção e Sistemas, área ergonomia, da Universidade Federal de Santa Catariana. Florianópolis, Brasil. Recuperado de https://repositorio.ufsc.br/ handle/123456789/89013
Gonçalves, D. M., Stein, A. T., \& Kapczinski, F. (2008). Avaliação de desempenho do Self-Reporting Questionnaire como instrumento de rastreamento psiquiátrico: Um estudo comparativo com o Structured Clinical Interview for DSM-IV-TR. Cadernos de Saúde Pública, 24(2), 380-390. Recuperado de http://www.lume.ufrgs.br/handle/10183/49591

Harmse, J. L., Engelbrecht, J. C. \& Bekker, J. L. (2016). The impact of physical and ergonomic hazards on poultry abattoir processing workers: A review. International Journal of Environmental Research and Public Health, 13(197). doi: 10.3390/ijerph13020197

Heck, F. M., \& Júnior, A. T. (2012). O trabalho degradado em frigoríficos e o adoecimento dos trabalhadores. VIII Seminário de Saúde do Trabalhador (em continuidade ao VII Seminário de Saúde do Trabalhador de Franca) e VI Seminário "O Trabalho em Debate". Anais. Franca-SP: UNESP/USP/STICF/CNTI/ UFSC. Recuperado de http://www.estudosdotrabalho.org/texto/gt6/o_trabalho.pdf.

Ikedo, F., \& Ruiz, R.C. (2015). Fazendo nexo entre a doença e o trabalho. Em F. Ikedo, F. Magajewski, J., Sardá, M. A., Espíndola, P. A. B., Oliveira \& R. C. Ruiz. Trabalhar e adoecer na agroindústria: da reabilitação à construção da Norma Regulamentadora dos Frigorificos (NR36) (p. 119-124). Florianópolis: Insular.

Instituto Fator Humano (2013). Relatório de Pesquisa: Perfil de Agravos à Saúde em Trabalhadores de Santa Catarina. [Mídia de gravação: CD]. Florianópolis: Instituto Fator Humano; UFSC, Univali, MPT, FECESC, FETIESC, SINTIARC, DIEESE.

Jacques, M. da G. (2007). O nexo causal em saúde/ doença mental no trabalho: Uma demanda para a psicologia. Psicologia \& Sociedade, 19(n. esp). Recuperado de http://www.scielo.br/pdf/psoc/ v19nspe/v19nspea15.pdf.

Krus, D. J., \& Helmstadter, G. C. (1993). The problem of negative reliabilities. Educational and Psychological Measurement, 53, 643-650. doi: 10.1177/0013164493053003005

Lander, L., Sorock, G.S., Smith, L. M., Stentz, T. L., Kim.S. S., Mittleman, M. A., \& Perry, M. J. (2015). Is depression a risk factor for meatpacking injuries? Journal of Work, (Preprint), 1-5. doi: 10.3233/ WOR-152147

Ludemir, A. B., \& Lewis, G. (2001). Links between social class and common mental disorders in Psico-USF, Bragança Paulista, v. 23, n. 3, p. 539-554, jul./set. 2018 
northeast Brazil. Social Psychiatric Epidemiology, 36, 101-107. doi: 10.1007/s001270050297

Pasquali, L. (2013). Psicometria: teoria dos testes na psicologia e na educaşão. 5 ed. Petrópolis: Vozes.

Pinetti, A. C. H., \& Buczek, M. D. R. (2015). Ergonomic work analysis in a brazilian poultry slaughterhouse cutting room. Proceedings of the 19th Triennial Congress of the IE $A$, Melbourne, 9-14. Recuperado de http://www.iea.cc/congress/2015/77.pdf

Santos, K. O. B., Araújo, T. M., \& Oliveira, N. F. de. (2009). Estrutura fatorial e consistência interna do Self-Reporting Questionnaire (SRQ-20) em população urbana. Cadernos de Saúde Pública, 25(1), 214-222. doi: 10.1590/S0102-311X2009000100023

Santos, K. O. B., Araújo, T. M., Pinho, P. S., \& Silva, A. C. C. (2010). Avaliação de um instrumento de mensuração de morbidade psíquica: Estudo de validação do Self-reporting questionnaire (SQR20). Revista Baiana de Saúde Pública, 34(3), 544-560. Recuperado de http://inseer.ibict.br/rbsp/index. $\mathrm{php} / \mathrm{rbsp} /$ article/viewFile/54/54
Sardá Junior, J., \& Ruiz, R. C. (2014). Resultados. Em F. Ikedo, F. Magajewski, J. Sardá, M. A. Espíndola, P. A. Oliveira, \& R. C. Ruiz. Trabalhar e adoecer na agroindústria: da reabilitação à construção da Norma Regulamentadora dos Frigorificos (NR36). (pp. 131-144). Florianópolis: Insular.

Walter, L. I. (2012). A saúde por um fio: Submissão voluntária de afastados de frigorificos de aves. Porto Alegre: Editora Abecer.

World Health Organization (1994). Expert Committee on Mental Health. User's Guide to Self Reporting Questionnaire (SRQ). Geneva: Author. Recuperado de http:/ / apps.who.int/iris/bitstream/10665/61113/1/ WHO_MNH_PSF_94.8.pdf

Zigmond, A. S., \& Snaith, R. P. (1983). The Hospital Anxiety and Depression Scale. Acta Psychiatrica Scandinavica, 67, 361-370. doi: 10.1111/ j.1600-0447.1983.tb09716.x

Recebido em: 02/06/2017 Reformulado em: 25/10/2017; 30/11/2017 Aprovado em: 13/12/2017 
Sobre os autores:

Romilda Guilland é psicóloga, especialista em Psicologia Clínica, mestre em Psicologia Clínica, com enfoque em saúde do trabalhador, doutora em Processos Psicossociais e de Saúde no Trabalho e nas Organizações, coordenadora da Comissão de Avaliação Psicológica do Conselho Regional de Psicologia - subsede de Cascavel - e Analista Judiciário, área Psicologia, do Tribunal de Justiça do Estado do Paraná.

E-mail: guil.ro@hotmail.com

ORCID: https://orcid.org/0000-0003-0858-7240

Roberto Moraes Cruz é especialista em avaliação Psicológica, Ergonomia e Psicologia Ocupacional, mestre em Psicologia e em Educação, doutor em Engenharia de Produção, pós-doutorado em Métodos e Diagnóstico, professor do Departamento de Psicologia da UFSC. Líder do Laboratório Fator Humano e pesquisador do Núcleo de Pesquisa em Neuropsicologia e Saúde.

E-mail: robertocruzdr@gmail.com

ORCID: https://orcid.org/0000-0003-4671-3498

Erikson Kaszubowski é doutor em Psicologia e psicólogo clínico da Universidade Federal de Santa Catarina. Desenvolve, ainda, pesquisas na elaboração de modelos probabilísticos para avaliação de intervenções psicológicas, com ênfase em modelagem bayesiana e técnicas computacionais baseadas em simulação.

E-mail: erikson.k@ufsc.br

ORCID: https://orcid.org/0000-0001-5481-1755

Contato com os autores:

Rua Dom Carmine Rocco, 595, Bairro Panceira

Toledo-PR, Brasil

CEP: 85902-270 